\title{
Basis Properties of Exponential Systems With Linear Phases in Morrey-Sobolev Type Spaces
}

\author{
Valid F. Salmanov ${ }^{1}$, Tarlan Z. Garayev ${ }^{1,2}$ and Saad R. El-Shabrawy ${ }^{3, *}$ \\ ${ }^{1}$ Institute of Mathematics and Mechanics, NAS of Azerbaijan, 9, B. Vahabzade St., Az1141, Baku, Azerbaijan \\ ${ }^{2}$ Khazar University, Department of Mathematics, 41, Mehseti St., Az1096, Baku , Azerbaijan \\ ${ }^{3}$ Faculty of Science, Damietta University, New Damietta, 34517, Egypt
}

Received: 3 Oct. 2016, Revised: 10 May 2017, Accepted: 15 May 2017

Published online: 1 Sep. 2017

\begin{abstract}
This paper is devoted to the study of basis properties of the system $\{t\} \cup\left\{e^{i(n+\beta \text { signn)t }}\right\}_{n \in Z}$, where $\beta$ is a real parameter, in Morrey-Sobolev-type spaces. We find sufficient conditions for the basicity in Morrey-Sobolev-type spaces in terms of inequalities of the parameter $\beta$.
\end{abstract}

Keywords: Morrey-Sobolev spaces, Basis properties.

\section{Introduction}

Morrey spaces were introduced by Morrey [1] in the setting of partial differential equations and appeared to be quite useful in the study of the local behavior of solutions of elliptic partial differential equations, a priori estimates and other topics in the theory of PDE. Precisely, it is a useful tool in the qualitative theory of elliptic differential equations [2,3]. Further, it provides a large class of examples of mild solutions of the Navier-Stokes system [4]. In the context of fluid dynamics, Morrey spaces have been used to model flow when vorticity is a singular measure supported on certain sets in $R^{n}$ [5]. There are sufficiently wide investigations related to fundamental problems in these spaces in view of differential equations, potential theory, maximal and singular operator theory and approximation theory (c.f. [6] and the references above). Special interest in the study of Morrey-type spaces arised in harmonic analysis and approximation theory $[7,8,9,10,11]$.

The splash of interest to Morrey-type spaces during the last decade allow to consider the basis properties of systems in such spaces in order to fill the gaps in the theory of Morrey spaces. The basis properties of exponential systems have been extensively investigated in various function spaces by several authors. Babenko [12] has proved that the degenerate system of exponentials $\left\{|t|^{\alpha} e^{i n t}\right\}_{n \in \mathbb{Z}}$ with $|\alpha|<\frac{1}{2}$ forms a basis for $L_{2}(-\pi, \pi)$ but does not form a Riesz basis when $\alpha \neq 0$, where $\mathbb{Z}$ is the set of integers. Generalization of this result to a more general degenerate function is given by Gaposhkin [13]. In [14], the conditions on the weight function $\rho$, for which the system $\left\{e^{i n t}\right\}_{n \in \mathbb{Z}}$ forms an unconditional basis for the weighted Besov space have been obtained. Similar problems have been studied in $[15,16]$. Basis properties of the systems of sines, cosines and exponentials with the linear phase in weighted Lebesgue space have been studied in $[17,18,19]$; see also $[20,21,22]$.

The basis properties of the exponential systems in Morrey-type spaces are much less studied. In the paper [23], there were obtained the basis properties of the system of exponentials in Morrey space. Also, in [24] the basis properties of the perturbed systems of exponentials in Morrey space have been investigated. On the other hand, the Riemann boundary value problem in Morrey-Hardy classes has been studied in [25].

In this paper we introduce a simple method for investigating the basis properties of the system $\{t\} \cup\left\{e^{i(n+\beta \text { signn }) t}\right\}_{n \in Z}$, where $\beta$ is a real parameter, in the Morrey-Sobolev space.

\footnotetext{
* Corresponding author e-mail: srshabrawy@yahoo.com
} 


\section{Preliminaries and Notation}

Throughout this paper, we always denote by $C$ a positive constant which is independent of main parameters, but it may have different values even in the same line. Denote the set of natural numbers by $\mathbb{N}$ and the set of nonnegative integers by $\mathbb{N}_{0}$. We always assume, unless otherwise stated, that $\alpha, p$ and $q$ are real numbers such that $0 \leq \alpha \leq 1,1<p<\infty$ and $p^{-1}+q^{-1}=1$.

Let $\Gamma$ be a rectifiable Jordan curve on the complex plane $\mathbb{C}$. By $|M|_{\Gamma}$ we denote the linear Lebesgue measure of a set $M \subset \Gamma$. The Morrey-Lebesgue (or simply, Morrey) space $L^{p, \alpha}(\Gamma)$ is the normed space of all measurable functions on $\Gamma$ equipped with the norm

$\|f\|_{L^{p, \alpha}(\Gamma)}=\left(\sup _{B} \frac{1}{|B \cap \Gamma|^{1-\alpha}} \int_{B \cap \Gamma}|f(t)|^{p}|d t|\right)^{\frac{1}{p}}<+\infty$,

where the supremum is taken over all disks $B$ centered on $\Gamma$. $L^{p, \alpha}(\Gamma)$ is a Banach space and $L^{p, 1}(\Gamma)=L_{p}(\Gamma)$, $L^{p, 0}(\Gamma)=L_{\infty}(\Gamma)$. The embedding $L^{p, \alpha_{1}}(\Gamma) \subset L^{p, \alpha_{2}}(\Gamma)$ is valid for $0 \leq \alpha_{1} \leq \alpha_{2} \leq 1$. Thus, $L^{p, \alpha}(\Gamma) \subset L_{p}(\Gamma)$ for $1<p<\infty$. The case of $\Gamma=[-\pi, \pi]$ will be denoted by $L^{p, \alpha}$.

Denote by $\tilde{L}^{p, \alpha}$ the linear subspace of $L^{p, \alpha}$ consisting of functions whose shifts are continuous in $L^{p, \alpha}$, i.e.

$\tilde{L}^{p, \alpha}=\left\{f \in L_{p, \alpha}:\|f(\cdot+\delta)-f(\cdot)\|_{L^{p, \alpha}} \rightarrow 0\right.$, as $\left.\delta \rightarrow 0\right\}$.

We always assume that a function $f$ continues $2 \pi$-periodically to the real axis $\mathbb{R}$. The closure of $\tilde{L}^{p, \alpha}$ in $L^{p, \alpha}$ will be denoted by $M L^{p, \alpha}$.

The Morrey-Sobolev space is denoted by $W_{p, \alpha}^{1}$ and consists of functions which belong, together with their derivatives of the first order, to the Morrey space $L^{p, \alpha}$ equipped with the norm

$$
\|f\|_{W_{p, \alpha}^{1}}=\|f\|_{L^{p, \alpha}}+\left\|f^{\prime}\right\|_{L^{p, \alpha}} .
$$

Denote by $\tilde{W}_{p, \alpha}^{1}$ the linear subspace of $W_{p, \alpha}^{1}$ consisting of functions whose shifts are continuous in $W_{p, \alpha}^{1}$. By $M W_{p, \alpha}^{1}$ we denote the closure of this space with respect to the norm (1). By $M \mathscr{L}_{p, \alpha}$ we denote the direct sum of $M L^{p, \alpha}$ and $\mathbb{C}(\mathbb{C}$ is the complex plane $)$

$$
M \mathscr{L}_{p, \alpha}=M L^{p, \alpha} \oplus \mathbb{C} .
$$

Let us define the norm in $M \mathscr{L}_{p, \alpha}$ in the following way

$$
\|\hat{u}\|_{M \mathscr{L}_{p, \alpha}}=\|u\|_{L^{p, \alpha}}+|\lambda|, \quad \hat{u}=(u ; \lambda) \in M \mathscr{L}_{p, \alpha} .
$$

We assume here some familiarity with basic concepts of basis theory and we refer to the book of Heil [26].

\section{The Main Result}

The main result reads

Theorem 1.Let $0<\alpha<1$. The system $\{t\} \cup\left\{e^{i(n+\beta \text { sign } n) t}\right\}_{n \in Z}$ forms a basis for $M W_{p, \alpha}^{1}(-\pi, \pi)$ if the following inequalities are satisfied

$$
-\frac{\alpha}{2 q} \leq \beta<\frac{\alpha}{2 p}
$$

The proof is based on constructing an isomorphism between the space $M W_{p, \alpha}^{1}$ and the space $M \mathscr{L}_{p, \alpha}$, which allows using the basis properties of the system $\left\{e^{i(n+\beta \text { signn }) t}\right\}_{n \in Z}$ in the space $M \mathscr{L}_{p, \alpha}$ presented in [24]. This will be shown in the following lemma.

Lemma 1.The operator $A: M \mathscr{L}_{p, \alpha} \longrightarrow M W_{p, \alpha}^{1}$, defined as $(A \hat{u})(t)=v(t)=\lambda+\int_{-\pi}^{t} u(\tau) d \tau$, is an isomorphism.

Proof.Firstly, let us show that the operator $A$ is well-defined. Indeed, since $L^{p, \alpha} \subset L_{p} \subset L_{1}$, then, for all $\hat{u} \in M \mathscr{L}_{p, \alpha}$ we have

$$
\begin{aligned}
\|A \hat{u}\|_{L^{p}, \alpha} & =\|v\|_{L^{p, \alpha}} \leq(2 \pi)^{\frac{\alpha}{p}}|\lambda|+\sup _{I \subset(-\pi, \pi)}\left\{\frac{1}{|I|^{1-\alpha}} \int_{I}\left|\int_{-\pi}^{t} u(\tau) d \tau\right|^{p} d t\right\}^{1 / p} \\
& \leq(2 \pi)^{\frac{\alpha}{p}}|\lambda|+\sup _{I \subset(-\pi, \pi)}\left\{\frac{1}{|I|^{1-\alpha}} \int_{I}\left(\int_{-\pi}^{\pi}|u(\tau)| d \tau\right)^{p} d t\right\}^{1 / p} \\
& =(2 \pi)^{\frac{\alpha}{p}}|\lambda|+(2 \pi)^{\frac{\alpha}{p}}\|u\|_{L_{1}(-\pi, \pi)}<+\infty .
\end{aligned}
$$

Additionally, $v^{\prime}=u \in L^{p, \alpha}$. Thus $A \hat{u} \in W_{p, \alpha}^{1}$. Next, we show that $A \hat{u} \in M W_{p, \alpha}^{1}$.

$$
\begin{aligned}
\|A \hat{u}(\cdot+\delta)-A \hat{u}(\cdot)\|_{W_{p, \alpha}^{1} \alpha} & \|v(\cdot+\delta)-v(\cdot)\|_{W_{p, \alpha}^{1}}=\|v(\cdot+\delta)-v(\cdot)\|_{L^{p}, \alpha} \\
& +\left\|v^{\prime}(\cdot+\delta)-v^{\prime}(\cdot)\right\|_{L^{p, \alpha}} \\
= & \left\|\int^{++\delta} u(\tau) d \tau\right\|_{L^{p, \alpha}}+\|u(\cdot+\delta)-u(\cdot)\|_{L^{p, \alpha}} \\
= & \sup _{I \subset(-\pi, \pi)}\left\{\frac{1}{|I|^{1-\alpha}} \int_{I}\left|\int_{t}^{t+\delta} u(\tau) d \tau\right|^{p} d t\right\}^{1 / p} \\
& +\|u(\cdot+\delta)-u(\cdot)\|_{L^{p}, \alpha} .
\end{aligned}
$$

By using the absolute continuity of the Lebesgue integral, when $t \in(-\pi, \pi)$, uniformly with respect to $t$, we have

$$
\int_{t}^{t+\delta} u(\tau) d \tau \rightarrow 0, \text { as } \delta \rightarrow 0
$$

Also, since $u \in M L^{p, \alpha}$, we have

$$
\|u(\cdot+\delta)-u(\cdot)\|_{L^{p, \alpha}} \rightarrow 0, \text { as } \delta \rightarrow 0 .
$$

Therefore

$$
\|A \hat{u}(\cdot+\delta)-A \hat{u}(\cdot)\|_{W_{p, \alpha}^{1}} \rightarrow 0, \text { as } \delta \rightarrow 0
$$


Furthermore, the operator $A$ is a bounded operator. Indeed, by using (3), we obtain that

$$
\|A \hat{u}\|_{W_{p, \alpha}^{1}} \leq(2 \pi)^{\frac{\alpha}{p}}|\lambda|+(2 \pi)^{\frac{\alpha}{p}}\|u\|_{L_{1}(-\pi, \pi)}+\|u\|_{L^{p, \alpha}} .
$$

Since

$$
\|u\|_{L_{1}} \leq C\|u\|_{L_{p}} \leq C\|u\|_{L^{p, \alpha}}
$$

we immediately have

$$
\|A \hat{u}\|_{W_{p, \alpha}^{1}} \leq C\left(|\lambda|+\|u\|_{L^{p, \alpha}}\right)=C\|\hat{u}\|_{M \mathscr{L}_{p, \alpha}}
$$

for an appropriate constant $C$. Next we show that $\operatorname{ker} A=\{0\}$. Let $A \hat{u}=0$, i.e. $\lambda+\int_{-\pi}^{t} u(\tau) d \tau=0$. If we differentiate both sides, we get $u(t)=0$, almost everywhere. Thus $\lambda=0$, and so $\hat{u}=0$. Finally, the operator $A$ is onto, since, for all $v \in M W_{p, \alpha}^{1}$, there exists $\hat{v}=\left(v^{\prime} ; v(-\pi)\right) \in M \mathscr{L}_{p, \alpha}$ and $A(\hat{v})=v$. Using the Banach theorem on inverse operators [27, Theorem 3.4], the inverse of $A$ is a continuous operator. This completes the proof of the lemma.

It is now easy to provide the

Proof(Proof of Theorem 1). It is known that with respect

to the condition (2), the system $\left\{e^{i(n+\beta \text { signn }) t}\right\}_{n \in Z}$ is a basis for $M L^{p, \alpha}$ [24, Corollary 3]. We will prove that the system $\left\{\hat{u}_{-1}\right\} \cup\left\{u_{0}\right\} \cup\left\{\hat{u}_{n}^{ \pm}\right\}, n \geq 1$ forms a basis for $M \mathscr{L}_{p, \alpha}$, where

$$
\begin{gathered}
\hat{u}_{-1}=\left(\begin{array}{c}
1 \\
-\pi
\end{array}\right), \hat{u}_{0}=\left(\begin{array}{c}
0 \\
1
\end{array}\right), \\
\hat{u}_{n}^{+}=\left(\begin{array}{c}
i(n+\beta) e^{i(n+\beta) t} \\
e^{-i(n+\beta) \pi}
\end{array}\right), \hat{u}_{n}^{-}=\left(\begin{array}{c}
-i(n+\beta) e^{-i(n+\beta) t} \\
e^{i(n+\beta) \pi}
\end{array}\right), n \geq 1 .
\end{gathered}
$$

Any element $\hat{u} \in M \mathscr{L}_{p, \alpha}$ has a unique expansion of the form

$$
\hat{u}=c_{-1} \hat{u}_{-1}+c_{0} \hat{u}_{0}+\sum_{n=1}^{\infty} c_{n}^{+} \hat{u}_{n}^{+}+\sum_{n=1}^{\infty} c_{n}^{-} \hat{u}_{n}^{-}
$$

This expansion is equivalent to the expansions

$$
\begin{gathered}
u(t)=c_{-1}+\sum_{n=1}^{\infty} i c_{n}^{+}(n+\beta) e^{i(n+\beta) t}+\sum_{n=1}^{\infty}(-i) c_{n}^{-}(n+\beta) e^{-i(n+\beta) t}, \\
\lambda=-\pi c_{-1}+c_{0}+\sum_{n=1}^{\infty} c_{n}^{+} e^{-i(n+\beta) \pi}+\sum_{n=1}^{\infty} c_{n}^{-} e^{i(n+\beta) \pi} .
\end{gathered}
$$

Following [24, Corollary 3] we obtain that (5) uniquely exists and belongs to the space $M L^{p, \alpha}$. Since $L^{p, \alpha} \subset L_{p}$, then, by using the main result of [28], for the system $\left\{e^{i(n+\beta \text { signn }) t}\right\}_{n \in Z}$, the Hausdorff-Young inequality holds for $\beta>-\frac{1}{2 q}$. That is, for $1<p \leq 2$, we have

$\left(\left|c_{-1}\right|^{q}+\sum_{n=1}^{\infty}\left|c_{n}^{-}(n+\beta)\right|^{q}+\sum_{n=1}^{\infty}\left|c_{n}^{+}(n+\beta)\right|^{q}\right)^{1 / q} \leq M\|u\|_{L_{p}}$,

where $\frac{1}{p}+\frac{1}{q}=1$. Applying Hölder's inequality, we obtain

$$
\begin{aligned}
& \left|c_{-1}\right|+\sum_{n=1}^{\infty}\left|c_{n}^{-}\right|+\sum_{n=1}^{\infty}\left|c_{n}^{+}\right|= \\
& \left|c_{-1}\right|+\sum_{n=1}^{\infty} \frac{1}{|n+\beta|}\left|(n+\beta) c_{n}^{-}\right|+\sum_{n=1}^{\infty} \frac{1}{\mid n+\beta) \mid}\left|(n+\beta) c_{n}^{+}\right| \\
& \leq\left|c_{-1}\right|+\sum_{n=1}^{\infty} \frac{1}{|n+\beta|^{p}} \sum_{n=1}^{\infty}\left|(n+\beta) c_{n}^{-}\right|^{q}+\sum_{n=1}^{\infty} \frac{1}{|n+\beta|^{p}} \sum_{n=1}^{\infty}\left|(n+\beta) c_{n}^{+}\right|^{q}<+\infty .
\end{aligned}
$$

Let us note that, when $n \geq 1$, we have $n+\beta \neq 0$. Therefore, with respect to the condition (2) the inequality $\beta>-\frac{1}{2}$ is valid.

For $p>2$, since $L^{p, \alpha} \subset L_{p} \subset L_{2}$, we have

$\left(\left|c_{-1}\right|^{2}+\sum_{n=1}^{\infty}\left|c_{n}^{-}(n+\beta)\right|^{2}+\sum_{n=1}^{\infty}\left|c_{n}^{+}(n+\beta)\right|^{2}\right)^{1 / 2} \leq M\|u\|_{L_{2}}$,

and similarly

$\left|c_{-1}\right|+\sum_{n=1}^{\infty}\left|c_{n}^{-}\right|+\sum_{n=1}^{\infty}\left|c_{n}^{+}\right| \leq\left|c_{-1}\right|+\sum_{n=1}^{\infty} \frac{1}{n+\left.\beta\right|^{2}}\left(\sum_{n=1}^{\infty}\left|(n+\beta) c_{n}^{-}\right|^{2}+\sum_{n=1}^{\infty}\left|(n+\beta) c_{n}^{+}\right|^{2}\right)<+\infty$.

So, we show that the series $\sum_{n=1}^{\infty}\left|c_{n}^{ \pm}\right|$is absolutely convergent. Therefore, in the series (6), the coefficient $c_{0}$ is uniquely defined. Thus, we have shown the existence and uniqueness of the expansion (4) for all $\hat{u} \in M \mathscr{L}_{p, \alpha}$. Thus, the system $\left\{\hat{u}_{-1}\right\} \cup\left\{\hat{u}_{0}\right\} \cup\left\{\hat{u}_{n}^{ \pm}\right\}_{n \geq 1}$ forms a basis for $M \mathscr{L}_{p, \alpha}$. We can easily show that, for the operator

$$
A \hat{u}=\lambda+\int_{-\pi}^{t} u(\tau) d \tau,
$$

the following relations are true

$A\left(\hat{u}_{-1}\right)=t, A\left(\hat{u}_{0}\right)=1, A\left(\hat{u}_{n}^{-}\right)=e^{-i(n+\beta) t}$, and $A\left(\hat{u}_{n}^{+}\right)=e^{i(n+\beta) t}$.

Since $A$ is an isomorphism, the system $\{t\} \cup\left\{e^{i(n+\beta \text { signn }) t}\right\}_{n \in Z}$ forms a basis for $M W_{p, \alpha}^{1}$. This completes the proof.

\section{Acknowledgment.}

The authors express their deep gratitude to Professor Bilal T. Bilalov, corresponding member of the National Academy of Sciences of Azerbaijan, for his inspiring guidance and valuable suggestions during the work. Also, the authors would like to express their indebtedness to the editor and the anonymous referees for their careful reading and making some useful comments which led to improvement of the presentation of the paper.

This work was supported by the Research Program Competition launched by the National Academy of Sciences of Azerbaijan (Program: Frame theory Applications of Wavelet Analysis to Signal Processing in Seismology and Other Fields). 


\section{References}

[1] C. B. Morrey, On the solutions of quasi-linear elliptic partial differential equations, Trans. Amer. Math. Soc., 43 (1938), 126-166.

[2] Y. Chen, Regularity of the solution to the Dirichlet problem in Morrey space, J. Partial Differ. Eqs. 15 (2002) 37-46.

[3] A. L. Mazzucato, Decomposition of Besov-Morrey spaces, in Harmonic Analysis at Mount Holyoke, Contemporary Mathematics 320 (2003), 279-294.

[4] P. G. Lemarie-Rieusset, Some remarks on the Navier-Stokes equations in $R^{3}$, J. Math. Phys. 39 (1988), 4108-4118.

[5] Y. Giga, T. Miyakawa, Navier-Stokes flow in $R^{3}$ with measures as initial vorticity and Morrey spaces, Comm. Partial Differential Equations 14 (1989), 577-618.

[6] J. Duoandikoetxea, Weight for maximal functions and singular integrals, NCTH Summer School on Harmonic Analysis in Taiwan, 2005.

[7] D. M. Israfilov, N. P. Tozman, Approximation by polynomials in Morrey-Smirnov classes, East J. Approx., 14 (2008), 255-269.

[8] D. M. Israfilov, N. P. Tozman, Approximation in MorreySmirnov classes, Azerbaijan J. Math. 1 (2011), 99-113.

[9] V. Kokilashvili, A. Meskhi, Boundedness of maximal and singular operators in Morrey spaces with variable exponent, Govern. College Univ. Lahore 72 (2008), 1-11.

[10] N. X. Ky, On approximation by trigonometric polynomials in $L_{p, u}$-spaces, Studia Sci. Math. Hungar 28 (1993), 183188.

[11] N. Samko, Weighted Hardy and singular operators in Morrey spaces, J. Math. Anal. Appl. 35 (1) (2009), 183-188.

[12] K. I. Babenko, On conjugate functions, Doklady Akademii Nauk SSSR 62 (2) (1948), 157-160.

[13] V. F. Gaposhkin, A generalization of the theorem of $M$. Riesz on conjugate functions, Mat. Sb. (N.S.) 46 (88), No. 3 (1958), 359-372

[14] K. S. Kazaryan and P. I. Lizorkin, Multipliers, bases and unconditional bases in the weighted spaces $B$ and $S B$, Trudy Math. Inst. Steklov. 187 (1989), 111-130; English transl. in Proc. Steklov Inst. Math. 1990, No. 3 (187).

[15] B. T. Bilalov, S. G. Veliev, On completeness of exponent system with complex coefficients in weighted spaces, Trans. Natl. Acad. Sci. Azerb. Ser. Phys.-Tech. Math. Sci. 25 (7) (2005), 9-14.

[16] B. T. Bilalov, S. G. Veliev, Bases of eigenfunctions of two discontinuous differential operators, Differ. Equ. 42 (10) (2006), 1503-1506.

[17] E. I. Moiseev, On basicity of the systems of cosines and sines in weighted space, Differ. Uravn. 34 (1) (1998), 40-44.

[18] E. I. Moiseev, The basicity in weighted space of a system of eigenfunctions of a differential operator, Differ. Uravn. 35 (2) (1999), 200-205.

[19] E. I. Moiseev, Basicity of the system of exponents, cosines and sines in $L_{p}$, Dokl. Akad. Nauk 275 (1984), 794-798.

[20] S. S. Pukhov, A. M. Sedletski, Bases of exponentials, sines and cosines in weighted spaces on a finite interval, Doklady Akademii Nauk. Rossijskaâ Akademiâ Nauk, 425 (4) (2009), 452-455.

[21] B. T. Bilalov, Basis of exponentials, cosines and sines that are eigenfunctions of differential operators, Differ. Equ. 39 (5) (2003), 652-657.

[22] B. T. Bilalov, On basicity of some systems of exponents, cosines and sines, Doklady Akademii Nauk. Rossijskaâ Akademiâ Nauk, 379 (2) (2001), 7-9.

[23] B. T. Bilalov, A. Guliyeva, On basicity of exponential systems in Morrey-type spaces, International Journal of Mathematics, 2012, Art. ID 184186, 12 pp.

[24] A. A. Huseynli, V. S. Mirzoyev, A. A. Quliyeva,On basicity of the perturbed system of exponents in Morrey-Lebesgue space, Azerb. J. Math. 7 (2) (2017), 197-216.

[25] B. T. Bilalov, T. B. Gasymov, A. A. Guliyeva, On solvability of Riemann boundary value problem in Morrey-Hardy classes. Turk. J. Math. 40 (5) (2016), 1085-1101.

[26] C. Heil, A Basis Theory Primer, Expanded Edition, Birkhäuser/Springer, New York, 2011.

[27] Y. M. Berezansky, Z. G. Sheftel, G. F. Us, Functional Analysis, Vol. I, Operator Theory: Advances and Applications, vol. 85, Birkhauser Verlag, Basel, 1996. Translated from the Russian original by Peter V. Malyshev.

[28] B. T. Bilalov, M. Kh. Karakash, The Hausdorff-Young type theorem for one system of exponents, Trans. of IMM of Azerb., 2004, vol. XXIV, No. 4, pp. 19-23.

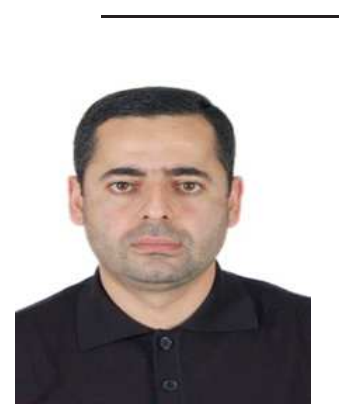

Valid F. Salmanov has graduated from Baku State University, Mechanics and Mathematics Faculty, Baku, Azerbaijan in 1995. $\mathrm{He}$ has obtained his $\mathrm{PhD}$ from Institute of Mathematics and Mechanics of NAS of Azerbaijan in 2006. He is an Associate Professor in the speciality of Differential Equations. His research interests include Mathematical Analysis, Differential Equations and Basis Theory.

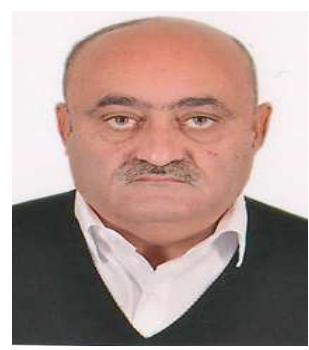

Basis Theory.
Tarlan Z. Garayev has graduated from Baku State University, Mechanics and Mathematics Faculty, Baku, Azerbaijan in 1990. $\mathrm{He}$ has obtained his $\mathrm{PhD}$ from Institute of Mathematics and Mechanics of NAS of Azerbaijan in 2014. His research interests include Differential Equations and 


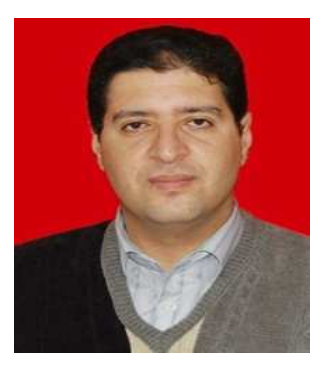

Saad R. El-Shabrawy

has obtained his B.Sc.

and M.Sc. from Mansoura

University (Damietta

Branch), Faculty of Science,

New Damietta, Egypt, in

2002 and 2007, respectively.

He has obtained his PhD

from Baku State University, Mechanics and Mathematics

Faculty, Baku, Azerbaijan in 2012. His research interests include Functional Analysis and Basis Theory. 